\title{
GROWTH RATE OF LIPSCHITZ CONSTANTS FOR RETRACTIONS BETWEEN FINITE SUBSET SPACES
}

\author{
EARNEST AKOFOR AND LEONID V. KOVALEV
}

\begin{abstract}
For any metric space $X$, finite subset spaces of $X$ provide a sequence of isometric embeddings $X=X(1) \subset X(2) \subset \cdots$. The existence of Lipschitz retractions $r_{n}: X(n) \rightarrow X(n-1)$ depends on the geometry of $X$ in a subtle way. Such retractions are known to exist when $X$ is an Hadamard space or a finite-dimensional normed space. But even in these cases it was unknown whether the sequence $\left\{r_{n}\right\}$ can be uniformly Lipschitz. We give a negative answer by proving that $\operatorname{Lip}\left(r_{n}\right)$ must grow with $n$ when $X$ is a normed space or an Hadamard space.
\end{abstract}

\section{INTRODUCTION}

Given a topological space $X$ and a positive integer $n$, the nonempty subsets of $X$ of cardinality at most $n$ form another topological space $X(n)$ with a natural quotient topology induced by the map that takes each ordered tuple $\left(x_{1}, \ldots, x_{n}\right)$ in the Cartesian product $X^{n}$ to the finite set $\left\{x_{1}, \ldots, x_{n}\right\}$ in $X(n)$. The space $X(n)$ is called the $n$th finite subset space of $X$ (the terms symmetric product or symmetric power are sometimes used as well). This concept goes back to Borsuk and Ulam [8]. When $X$ is a metric space, $X(n)$ becomes a metric space with respect to Hausdorff distance which is given by

$$
\begin{aligned}
& d_{H}\left(\left\{x_{1}, \ldots, x_{n}\right\},\left\{x_{1}^{\prime}, \ldots, x_{n}^{\prime}\right\}\right) \\
& :=\max \left\{\max _{i} \min _{j} d\left(x_{i}, x_{j}^{\prime}\right), \max _{i} \min _{j} d\left(x_{j}, x_{i}^{\prime}\right)\right\} .
\end{aligned}
$$

See, e.g., [2, Proposition 1.2.2] for details of this metrization. The natural embeddings $X=X(1) \subset X(2) \subset \cdots$ are isometric with respect to the Hausdorff distance.

If $X$ and $Y$ are metric spaces, a map $f: X \rightarrow Y$ is called Lipschitz if there is a number $L \geq 0$ such that $d\left(f(x), f\left(x^{\prime}\right)\right) \leq L d\left(x, x^{\prime}\right)$ for all $x, x^{\prime} \in X$. The least of such numbers $L$ is denoted by $\operatorname{Lip}(f)$ and is called the Lipschitz constant of $f$. If $Y \subset X$, a map $r: X \rightarrow Y$ is a retraction if its restriction to $Y$ is the identity map. If $r$ is in addition Lipschitz, it is called a Lipschitz retraction.

2020 Mathematics Subject Classification. Primary 54E40; Secondary 46B20, 54B20, 54C15.

Key words and phrases. normed space, metric space, Hadamard space, finite subset space, Lipschitz retraction.

L.V.K. supported by the National Science Foundation grant DMS-1764266. 
A Lipschitz retraction $X(n) \rightarrow X(k)$, where $k<n$, can be interpreted as a robust choice of $k$ clusters within a finite set. Indeed, given a set $A \subset X$ of cardinality $|A| \leq n$, we must choose a set $r(A)$ with $|r(A)| \leq k$ subject to the conditions that $r(A)=A$ if $|A| \leq k$, and $r(A)$ a Lipschitz function of $A$.

Some spaces $X$ present topological obstructions to the existence of such retractions. For example, if $X$ is the circle $S^{1}$, then $X(3)$ is homeomorphic to 3 -sphere [9] and cannot be retracted onto $X(1)=S^{1}$, being simply-connected. Section 2 presents a more general obstruction of this type. If $X$ is a Hilbert space of any dimension (finite or infinite), for every $n$ there exists a Lipschitz retraction $r_{n}: X(n) \rightarrow X(n-1)$ with $\operatorname{Lip}\left(r_{n}\right) \leq \max \left(n^{3 / 2}, 2 n-1\right)$ [15. (2.5)]. Question 3.2 in [15] and Remark 3.5 in [5] asked whether $\operatorname{Lip}\left(r_{n}\right)$ can be bounded independently of $n$. Our first result shows that it must grow at least linearly with respect to $n$, provided that $\operatorname{dim} X \geq 2$.

Theorem 1.1. Let $X$ be a normed space over $\mathbb{R}$ with $\operatorname{dim} X \geq 2$. Suppose that $r: X(n) \rightarrow$ $X(k)$ is a Lipschitz retraction, where $1 \leq k \leq n-1$. Then

$$
\operatorname{Lip}(r) \geq \frac{k n}{2 \pi(n-1)}-\frac{1}{2} \text {. }
$$

Moreover, if $X$ is a Hilbert space, then

$$
\operatorname{Lip}(r) \geq \frac{k n}{\pi(n-1)}-1
$$

The case $\operatorname{dim} X=1$, when $X$ is isometric to $\mathbb{R}$, is covered by our second main theorem. It concerns Hadamard spaces, which share the geometric properties of Hilbert spaces but not necessarily their linear structure. To define them, we need the notion of a geodesic space.

A geodesic in a metric space $(X, d)$ is a mapping $\gamma:[0,1] \rightarrow X$ such that for all $t, s \in[0,1]$ we have $d(\gamma(t), \gamma(s))=|t-s| d(\gamma(0), \gamma(1))$. In geometric terms, a geodesic is a curve parametrized proportionally to its arclength. If for any two points $p, q \in X$ there exists a geodesic with $\gamma(0)=p$ and $\gamma(1)=q$, then $X$ is called a geodesic space.

Definition 1.2. A complete geodesic space is called an Hadamard space if for every point $z$ and every geodesic $\gamma$ we have

$$
d(\gamma(t), z)^{2} \leq(1-t) d(\gamma(0), z)^{2}+t d(\gamma(1), z)^{2}-t(1-t) d(\gamma(0), \gamma(1))^{2}
$$

for all $t \in[0,1]$.

We refer to [4] for equivalent definitions and the motivation behind the concept of an Hadamard space. Theorem 3.2 in [5] asserts that for every Hadamard space $X$ and every $n \geq 2$ there exists a Lipschitz retraction $r_{n}: X(n) \rightarrow X(n-1)$ with $\operatorname{Lip}\left(r_{n}\right) \leq$ 
$\max \left(2 n^{2}+\sqrt{n}, 4 n^{3 / 2}+1\right)$. The following theorem gives a lower bound for $\operatorname{Lip}\left(r_{n}\right)$, answering a question posed in [5, Remark 3.5].

Theorem 1.3. Let $X$ be either a normed space over $\mathbb{R}$ of dimension $\operatorname{dim} X \geq 1$, or an Hadamard space containing more than one point. If $r: X(n) \rightarrow X(n-1)$ is a Lipschitz retraction, then $\operatorname{Lip}(r) \geq n-3$.

The paper is organized as follows. In Section 2 we collect the necessary results from the algebraic topology of finite subset spaces. Section 3 contains preliminary results on the properties of Lipschitz retractions. Theorems 1.1 and 1.3 are proved in sections 4 and 5 , respectively. Corollary 5.2 provides a more general version of Theorem 1.3 . Note that Theorem 1.3 gives a slightly better lower bound than Theorem 1.1. On the other hand, Theorem 1.1 applies to retractions onto $X(k)$ for any $k<n$, not only $k=n-1$.

\section{Topology of Finite SUbSET SPACES}

Let $H_{n}(X)$ denote the $n$th singular homology group of a topological space $X$ [11, p. 108]. The homology groups of finite subset spaces $S^{1}(n)$ were computed by $\mathrm{Wu}[18$ ] and their homotopy type was determined by Tuffley [17, see also [10].

Theorem 2.1. ([18, Theorem III], [17, Theorems 4-5]). Given $n \in \mathbb{N}$, let $m$ be the largest odd integer not exceeding $n$. The homology groups $H_{0}\left(S^{1}(n)\right)$ and $H_{m}\left(S^{1}(n)\right)$ are isomorphic to $\mathbb{Z}$, and all other homology groups of $S^{1}(n)$ are trivial. Moreover, when $n$ is odd, the inclusion $S^{1}(n) \subset S^{1}(n+1)$ induces the doubling map $k \mapsto 2 k$ on the homology group $H_{n}\left(S^{1}(n)\right)$.

The homology presents an obstruction to the existence of continuous retractions between the finite subset spaces of the circle.

Proposition 2.2. If $1 \leq k \leq n-1$, there is no continuous retraction $S^{1}(n) \rightarrow S^{1}(k)$.

Proof. Suppose there exists a continuous retraction $r: S^{1}(n) \rightarrow S^{1}(k)$. The map induced by $r$ on the homology groups of $S^{1}(n)$ is a left inverse of the map induced by the inclusion of $S^{1}(k)$ into $S^{1}(n)$ [11, p. 111]. In particular, the latter map is injective.

Let $m$ be the greatest odd integer not exceeding $k$. Since $H_{m}\left(S^{1}(k)\right)$ is isomorphic to $\mathbb{Z}$, the group $H_{m}\left(S^{1}(n)\right)$ must be nontrivial. Theorem 2.1 implies that $m$ is the greatest odd integer not exceeding $n$. This is only possible if $n$ is even and $k=m=n-1$. However, in this case the inclusion of $S^{1}(n-1)$ into $S^{1}(n)$ induces the doubling map on the homology groups $H_{n-1}$, and this map does not have a left inverse, contradicting the previous paragraph. 
The obstruction presented by Proposition 2.2 is the basis of our proof of Theorem 1.1. To prove Theorem 1.3 we need to find some topological obstruction within the subsets of $\mathbb{R}$. It is provided by pinned finite subset spaces, which are defined as follows.

Definition 2.3. Given a set $U \in X(n)$, the $U$-pinned finite subset space of $X$ is

$$
X(n, U):=\{A \in X(n): U \subset A\} .
$$

The space $X(n, U)$ is empty when $n<|U|$. Notable examples of pinned finite subset spaces include

$$
\mathcal{D}^{n}:=I(n+2,\{0,1\})
$$

where $I$ is the interval $[0,1]$ and $n=0,1, \ldots$ The studies of $\mathcal{D}^{n}$ go back to Schori [16] who proved that $I(n)$ is a double cone of $\mathcal{D}^{n-2}$ when $n \geq 2$. Andersen, Marjanović and Schori [3] called the spaces $\mathcal{D}^{n}$ with even $n$ "higher-dimensional dunce hats" because $\mathcal{D}^{2}$ is homeomorphic to the "dunce hat" space introduced by Zeeman [19] and $\mathcal{D}^{n}$ shares some features of $\mathcal{D}^{2}$ when $n$ is even.

Theorem 2.4. [3, Theorem 3.4]. When $n$ is even, $\mathcal{D}^{n}$ is contractible. When $n$ is odd, $\mathcal{D}^{n}$ has the homotopy type of $S^{n}$.

As with the ordinary finite subset spaces, we have natural inclusions $X(k, U) \subset X(n, U)$ when $|U| \leq k \leq n$. For example, $\mathcal{D}^{0} \subset \mathcal{D}^{1} \subset \cdots$.

Corollary 2.5. When $n$ is even, there is no continuous retraction of $\mathcal{D}^{n}$ onto $\mathcal{D}^{n-1}$.

Proof. By Theorem 2.4, the homology group $H_{n-1}\left(\mathcal{D}^{n-1}\right)$ is isomorphic to $\mathbb{Z}$ while $H_{n-1}\left(\mathcal{D}^{n}\right)$ is trivial. Since the former group does not embed in the latter, $\mathcal{D}^{n-1}$ is not a retract of $\mathcal{D}^{n}$.

Corollary 2.5 is a less complete result than Proposition 2.2. We do not know if $\mathcal{D}^{n}$ retracts onto $\mathcal{D}^{n-1}$ when $n$ is odd. An interesting related question is whether each higherdimensional dunce hat $\mathcal{D}^{2 k}$ is an absolute Lipschitz retract, meaning that it is a Lipschitz retract in any metric space that contains it. At present it is not known whether finite subset spaces inherit the absolute Lipschitz retract property: see [1, 2, 14] for partial results.

Another example of a pinned finite subset space is $S^{1}(n,\{1\})$ which was studied by Tuffley [17, p. 1131]. This space is homeomorphic to $\mathcal{D}^{n-1}$. More specificially, the map $A \mapsto\left\{e^{2 \pi i t}: t \in A\right\}$ is a bi-Lipschitz homeomorphism of $\mathcal{D}^{n-1}$ onto $S^{1}(n,\{1\})$ : see the proof of Theorem 3.1 in [7]. 


\section{Properties of Lipschitz Retractions}

Let $X$ be a metric space and $n \geq 2$. The minimum separation function $\delta_{n}: X(n) \rightarrow[0, \infty)$ is defined as follows:

$$
\delta_{n}(A)= \begin{cases}\min \left\{d_{X}(p, q): p, q \in A, p \neq q\right\} & \text { if }|A|=n \\ 0 & \text { if }|A|<n\end{cases}
$$

The importance of $\delta_{n}$ stems from the following observation.

Lemma 3.1. Suppose $X$ is a metric space and $n \geq 2$. For each $A \in X(n)$ there is $B \in X(n-1)$ such that $d_{H}(A, B) \leq \delta_{n}(A)$. If, in addition, $X$ is a geodesic space, then the conclusion can be strengthened to $d_{H}(A, B) \leq \frac{1}{2} \delta_{n}(A)$.

Proof. If $|A|<n$, the set $B=A$ satisfies the conclusion. Suppose $|A|=n$. Let $p, q \in A$ be two points such that $d_{X}(p, q)=\delta_{n}(A)$. Then the set $B=A \backslash\{p\}$ has $n-1$ elements and $d_{H}(A, B) \leq \delta_{n}(A)$.

If $X$ is a geodesic space, let $m$ be the midpoint of a geodesic from $p$ to $q$ and define $B=(A \cup\{m\}) \backslash\{p, q\}$. This set has $n-1$ elements and $d_{H}(A, B) \leq \frac{1}{2} \delta_{n}(A)$.

Lemma 3.2. Let $X$ be a metric space and $1 \leq k<n$. Suppose $r: X(n) \rightarrow X(k)$ is a Lipschitz retraction. Then for every $A \in X(n)$ we have

$$
d_{H}(r(A), A) \leq(\operatorname{Lip}(r)+1) \operatorname{dist}_{H}(A, X(k))
$$

where $\operatorname{dist}_{H}(A, X(k))=\inf \left\{d_{H}(A, B): B \in X(k)\right\}$.

Proof. For every $B \in X(k)$ we have $r(B)=B$, hence

$$
d_{H}(r(A), B)=d_{H}(r(A), r(B)) \leq \operatorname{Lip}(r) d_{H}(A, B) .
$$

By the triangle inequality, $d_{H}(r(A), A) \leq(\operatorname{Lip}(r)+1) d_{H}(A, B)$. Taking the infimum over $B \in X(k)$ yields (3.2).

Lemma 3.3. Let $X$ be a metric space such that there exists a Lipschitz retraction $r: X(n) \rightarrow$ $X(k)$ for some integers $1 \leq k<n$. Suppose $Y$ is a metric space such that there exist Lipschitz maps $f: X \rightarrow Y$ and $g: Y \rightarrow X$ with the property $f \circ g=\operatorname{id}_{Y}$. Then there exists a Lipschitz retraction $s: Y(n) \rightarrow Y(k)$ with $\operatorname{Lip}(s) \leq \operatorname{Lip}(f) \operatorname{Lip}(g) \operatorname{Lip}(r)$.

Proof. The map $g$ induces a map $g_{n}: Y(n) \rightarrow X(n)$ such that $g_{n}(A)$ is the image of set $A$ under $g$. From the definition (1.1) of Hausdorff distance it is easy to see that $\operatorname{Lip}\left(g_{n}\right)=$ $\operatorname{Lip}(g)$. Similarly, $f$ induces a map $f_{k}: X(k) \rightarrow Y(k)$. Let $s=f_{k} \circ r \circ g_{n}$. By construction, $s$ maps $Y(n)$ to $Y(k)$ and has $\operatorname{Lipschitz}$ constant at most $\operatorname{Lip}(f) \operatorname{Lip}(g) \operatorname{Lip}(r)$. If $A \in Y(k)$ then $g_{n}(A) \in X(k)$, hence $s(A)=f_{k}\left(g_{n}(A)\right)=A$ by the property $f \circ g=\operatorname{id}_{Y}$. Thus $s$ is a Lipschitz retraction onto $Y(k)$. 
Two useful special cases of Lemma 3.3 are: (a) $Y$ is a Lipschitz retract of $X$, with $f$ being the inclusion map; (b) $Y$ is bi-Lipschitz equivalent to $X$, with $g=f^{-1}$.

\section{Normed SPACES: PROOF OF THEOREM 1.1}

Let $X$ be a normed space over $\mathbb{R}$ of dimension at least 2 . The following statement is a special case of [6, Proposition G.1] which summarizes the results of John [12] and KadetsSnobar [13].

Lemma 4.1. Let $Z$ be a 2-dimensional subspace of a normed space $X$. Then there exists a linear projection $P: X \rightarrow Z$, and a linear isomorphism $T: \mathbb{R}^{2} \rightarrow Z$ such that $\|P\| \leq \sqrt{2}$, $\|T\| \leq \sqrt{2}$, and $\left\|T^{-1}\right\| \leq 1$.

Lemma 4.1 leads us to consider the geometry of finite subsets of $\mathbb{R}^{2}$ which is the subject of the following lemma.

Lemma 4.2. Let $S^{1} \subset \mathbb{R}^{2}$ be the unit circle centered at 0 , equipped with the arclength metric. For any set $A \in S^{1}(n)$ and any $k \in 1, \ldots, n-1$ there exists $B \in S^{1}(k)$ such that $d_{H}(A, B) \leq \pi(n-1) /(k n)$.

Proof. Let $A_{j}$ be the result of rotating $A$ by the angle $2 \pi j / k$, and let $R=\bigcup_{j=1}^{k} A_{j}$. Since $R$ has at most $k n$ points, its complement in $S^{1}$ contains an open arc of length $2 \pi /(k n)$. The $k$-fold symmetry of $R$ implies that it is covered by $k$ uniformly spaced closed arcs $\Gamma_{1}, \ldots, \Gamma_{k} \subset S^{1}$ of length

$$
\frac{2 \pi}{k}-\frac{2 \pi}{k n}=\frac{2 \pi(n-1)}{k n}
$$

Therefore, $A \subset \bigcup_{j=1}^{k} \Gamma_{j}$. Let $B$ be the set of midpoints of all $\operatorname{arcs} \Gamma_{j}$ such that $\Gamma_{j} \cap A$ is nonempty. Then every point of $B$ is within distance at most $\pi(n-1) /(k n)$ of some point of $A$, and vice versa. Since $|B| \leq k$, the lemma is proved.

The estimate in Lemma 4.2 is best possible when $k=n-1$, as one can check using a set $A$ of $n$ equally spaced points. Lemma 4.2 also applies when $S^{1}$ is equipped with chordal metric, i.e. the restriction of the Euclidean metric on $\mathbb{R}^{2}$, because the chordal metric is majorized by arclength.

Proof of Theorem 1.1. Let $Y=\mathbb{R}^{2}$ and let $Z, P, T$ be as in Lemma 4.1. The mappings $f=T^{-1} \circ P$ and $g=T$ satisfy the assumptions of Lemma 3.3. Therefore, there exists a retraction $s: Y(n) \rightarrow Y(k)$ with $\operatorname{Lip}(s) \leq 2 \operatorname{Lip}(r)$. When $X$ is a Hilbert space, this can be improved to $\operatorname{Lip}(s) \leq \operatorname{Lip}(r)$ because any two-dimensional subspace $Z \subset X$ is isometric to $\mathbb{R}^{2}$ and $P$ can be the orthogonal projection onto $Z$. Therefore, both cases of Theorem 1.1 
will be proved if we show that $\operatorname{Lip}(s) \geq \frac{k n}{\pi(n-1)}-1$. Suppose, toward a contradiction, that the constant $c:=\frac{\pi(n-1)}{k n}(\operatorname{Lip}(s)+1)$ satisfies $c<1$.

Let $S^{1} \subset \mathbb{R}^{2}$ be the unit circle centered at 0. From Lemmas 3.2 and 4.2 it follows that $d_{H}(s(A), A) \leq c$ for every $A \in S^{1}(n)$. Hence $s(A)$ is contained in the set $W=\{x \in$ $\left.\mathbb{R}^{2}:\|x\| \geq 1-c\right\}$. The radial projection $f(x)=x /\|x\|$ provides a Lipschitz retraction of $W$ onto $S^{1}$. Therefore, the mapping $A \mapsto f(s(A))$ is a Lipschitz retraction on $S^{1}(n)$ onto $S^{1}(k)$. This contradicts Proposition 2.2 ,

\section{Metric SPACES: PROOF OF THEOREM 1.3}

We begin with the special case of retractions between the finite subset spaces of an interval on the real line. The definition of the Hausdorff distance (1.1) implies that for any two nonempty finite sets $A, B \subset \mathbb{R}$ we have

$$
|\max A-\max B| \leq d_{H}(A, B), \quad|\min A-\min B| \leq d_{H}(A, B) .
$$

Theorem 5.1. Let $I=[a, b] \subset \mathbb{R}$ where $-\infty<a<b<\infty$. If $r: I(n) \rightarrow I(n-1)$ is a Lipschitz retraction, then

$$
\operatorname{Lip}(r) \geq \begin{cases}n-2, & n \text { is even } \\ n-3, & n \text { is odd }\end{cases}
$$

Proof. The choice of the interval $[a, b]$ does not matter in this theorem. Indeed, if $\phi: \mathbb{R} \rightarrow \mathbb{R}$ is an invertible affine transformation, then $A \mapsto \phi\left(r\left(\phi^{-1}(A)\right)\right)$ is a Lipschitz retraction between the finite subset spaces of $\phi(I)$, with the same Lipschitz constant Lip $(r)$. Thus, we can choose any convenient interval $I$ in the proof, and we use two different intervals for the two cases that follow.

Case 1: $n$ is even. Assume $n \geq 4$ since the statement is trivial for $n=2$. Let $I=[0,1]$. Toward a contradiction, suppose that the quantity $c:=(\operatorname{Lip}(r)+1) /(n-1)$ satisfies $c<1$. Recall the subsets $\mathcal{D}^{n-2} \subset I(n)$ defined by (2.1). For every set $A \in \mathcal{D}^{n-2}$ the minimal separation (3.1) satisfies $\delta_{n}(A) \leq 1 /(n-1)$. From Lemmas 3.1 and 3.2 it follows that

$$
d_{H}(r(A), A) \leq \frac{1}{2(n-1)}(\operatorname{Lip}(r)+1) \leq c / 2 .
$$

By (5.1) we have $\min r(A) \leq c / 2$ and $\max r(A) \geq 1-c / 2$. For $t \in[0,1]$ let

$$
f_{A}(t)=\frac{t-\min r(A)}{\max r(A)-\min r(A)} .
$$

Since the denominator in (5.3) is bounded below by $1-c$, the function $f_{A}$ is $(1-c)^{-1}$ Lipschitz with respect to $t$. It is also Lipschitz continuous with respect to $A$ by virtue of (5.1). Therefore, the mapping $s(A):=f_{A}(r(A))$ is Lipschitz continuous on $\mathcal{D}^{n-2}$. By construction, the set $s(A)$ consists of at most $n-1$ points, $\min s(A)=0$, and $\max s(A)=1$. 
Thus $s(A) \in \mathcal{D}^{n-3}$. If $|A|<n$ then $r(A)=A$, hence $s(A)=A$. We have proved that $s$ is a Lipschitz retraction of $\mathcal{D}^{n-2}$ onto $\mathcal{D}^{n-3}$, which contradicts Corollary 2.5.

Case 2: $n$ is odd. We may assume $n \geq 5$ since the statement is trivial for $n \leq 3$. Let $I=$ $[0,2]$. Toward a contradiction, suppose that the quantity $c:=(\operatorname{Lip}(r)+1) /(n-2)$ satisfies $c<1$. Let $Y=[0,1] \cup\{2\}$ and consider the pinned finite subset space $\mathcal{E}=Y(n,\{0,1,2\})$. For every set $A \in \mathcal{E}$, all but one of its points lie in $[0,1]$. Hence its minimal separation satisfies $\delta_{n}(A) \leq 1 /(n-2)$. By Lemmas 3.1 and 3.2 we have

$$
d_{H}(r(A), A) \leq \frac{1}{2(n-2)}(\operatorname{Lip}(r)+1) \leq c / 2 .
$$

Since $\{0,1,2\} \subset A$, it follows that $r(A)$ meets each of the intervals $[0, c / 2],[1-c / 2,1+c / 2]$, and $[2-c / 2,2]$. Moreover, $r(A)$ is disjoint from the interval $(1+c / 2,2-c / 2)$.

Let

$$
s(A)=r(A) \cap[0,1+c / 2]=r(A) \cap[0,2-c / 2) .
$$

Note that $|s(A)| \leq r(A)-1 \leq n-2$ since $r(A)$ meets $[2-c / 2,2]$. Suppose that $A, B \in \mathcal{E}$ are such that $\Delta:=d_{H}(r(A), r(B))<1-c$. By the definition of $d_{H}$, for every $a \in s(A)$ there exists $b \in r(B)$ such that $|a-b| \leq \Delta$. Then $b \leq(1+c / 2)+\Delta<2-c / 2$, which implies $b \in s(B)$. In conclusion,

$$
d_{H}(s(A), s(B)) \leq d_{H}(r(A), r(B))
$$

whenever the right hand side is less than $1-c$. Since $r$ is Lipschitz continuous, (5.4) shows that $s$ is also Lipschitz continuous.

For $t \in[0,1]$ let

$$
f_{A}(t)=\frac{t-\min s(A)}{\max s(A)-\min s(A)}
$$

and note that the denominator is bounded below by $1-c$. As in Case 1 , it follows that $f_{A}(t)$ is Lipschitz with respect to both $t$ and $A$. Therefore, the mapping $\sigma(A):=f_{A}(s(A))$ is Lipschitz continuous on $\mathcal{E}$. By construction, the set $\sigma(A)$ consists of at most $n-2$ points, $\min \sigma(A)=0$, and $\max \sigma(A)=1$. Thus, $\sigma(A) \in \mathcal{D}^{n-4}$. If $A \in \mathcal{E}$ has fewer than $n$ points, then $r(A)=A$, hence $\sigma(A)=A \cap[0,1]$.

The space $\mathcal{D}^{n-3}$ is isometric to $\mathcal{E}$ via the map $\iota(A)=A \cup\{2\}$. The previous paragraph shows that the composition $\sigma \circ \iota$ is a Lipschitz retraction of $\mathcal{D}^{n-3}$ onto $\mathcal{D}^{n-4}$. Since $n-3$ is even, we have a contradiction with Corollary 2.5.

The following statement is an immediate consequence of Theorem 5.1 and Lemma 3.3 .

Corollary 5.2. Suppose $X$ is a metric space. Fix an integer $n$ such that there exists a Lipschitz retraction $r: X(n) \rightarrow X(n-1)$. Suppose $I \subset \mathbb{R}$ is a nondegenerate compact 
interval and there exist Lipschitz maps $f: X \rightarrow I$ and $g: I \rightarrow X$ such that $f \circ g=\mathrm{id}_{I}$. Then

$$
\operatorname{Lip}(r) \geq \frac{n-3}{\operatorname{Lip}(f) \operatorname{Lip}(g)}
$$

Proof of Theorem 1.3. Suppose $r: X(n) \rightarrow X(n-1)$ is a Lipschitz retraction.

Case 1: $X$ is a normed space. Let $I=[0,1]$. Pick a unit vector $u \in X$ and its norming functional $\varphi \in X^{*}$, that is, a linear functional $\varphi: X \rightarrow \mathbb{R}$ such that $\|\varphi\|_{X^{*}}=1$ and $\varphi(u)=1$. The existence of such $\varphi$ follows from the Hahn-Banach theorem. Define $g: I \rightarrow X$ by $g(t)=t u$, and $f: X \rightarrow I$ by $f(x)=\min (\max (\varphi(x), 0), 1)$. Note that both $f$ and $g$ are 1-Lipschitz and $f \circ g=\operatorname{id}_{I}$. By Corollary 5.2 we have $\operatorname{Lip}(r) \geq n-3$.

Case 2: $X$ is an Hadamard space. Pick any two distinct points $p, q \in X$ and let $I=\left[0, d_{X}(p, q)\right]$. Since Hadamard spaces are geodesic, there exists an isometric embedding $g: I \rightarrow X$, namely a reparametrized geodesic connecting $p$ to $q$. Since $g(I)$ is a closed convex subset of $X$, the nearest-point projection onto $X$ is a 1-Lipschitz map [4, Theorem 2.1.12]. Let $f$ be the composition of this projection with $g^{-1}$. Since $\operatorname{Lip}(f)=1=\operatorname{Lip}(g)$, Corollary 5.2 yields $\operatorname{Lip}(r) \geq n-3$ as claimed.

\section{REFERENCES}

[1] Earnest Akofor. On Lipschitz retraction of finite subsets of normed spaces. Israel J. Math., 234(2):777808, 2019.

[2] Earnest Akofor. Metric geometry of finite subset spaces. PhD thesis, Syracuse University, 2020. https://arxiv.org/abs/2004.09419.

[3] Robert N. Andersen, M. M. Marjanović, and Richard M. Schori. Symmetric products and higherdimensional dunce hats. Topology Proc., 18:7-17, 1993.

[4] Miroslav Bačák. Convex analysis and optimization in Hadamard spaces, volume 22 of De Gruyter Series in Nonlinear Analysis and Applications. De Gruyter, Berlin, 2014.

[5] Miroslav Bačák and Leonid V. Kovalev. Lipschitz retractions in Hadamard spaces via gradient flow semigroups. Canad. Math. Bull., 59(4):673-681, 2016.

[6] Yoav Benyamini and Joram Lindenstrauss. Geometric nonlinear functional analysis. Vol. 1, volume 48 of American Mathematical Society Colloquium Publications. American Mathematical Society, Providence, RI, 2000.

[7] Marina Borovikova, Zair Ibragimov, and Hassan Yousefi. Symmetric products of the real line. J. Anal., 18:53-67, 2010.

[8] Karol Borsuk and Stanislaw Ulam. On symmetric products of topological spaces. Bull. Amer. Math. Soc., 37(12):875-882, 1931.

[9] Raoul Bott. On the third symmetric potency of $S_{1}$. Fund. Math., 39:264-268 (1953), 1952.

[10] Naotsugu Chinen and Akira Koyama. On the symmetric hyperspace of the circle. Topology Appl., 157(17):2613-2621, 2010.

[11] Allen Hatcher. Algebraic topology. Cambridge University Press, Cambridge, 2002.

[12] Fritz John. Extremum problems with inequalities as subsidiary conditions. In Studies and Essays Presented to R. Courant on his 60th Birthday, January 8, 1948, pages 187-204. Interscience Publishers, Inc., New York, N. Y., 1948.

[13] M. I. Kadec and M. G. Snobar. Certain functionals on the Minkowski compactum. Mat. Zametki, 10:453-457, 1971. 
[14] Leonid V. Kovalev. Symmetric products of the line: embeddings and retractions. Proc. Amer. Math. Soc., 143(2):801-809, 2015.

[15] Leonid V. Kovalev. Lipschitz retraction of finite subsets of Hilbert spaces. Bull. Aust. Math. Soc., 93(1):146-151, 2016.

[16] Richard M. Schori. Hyperspaces and symmetric products of topological spaces. Fund. Math., 63:77-88, 1968.

[17] Christopher Tuffley. Finite subset spaces of $S^{1}$. Algebr. Geom. Topol., 2:1119-1145, 2002.

[18] Wen-Tsün Wu. Note sur les produits essentiels symétriques des espaces topologiques. C. R. Acad. Sci. Paris, 224:1139-1141, 1947.

[19] E. C. Zeeman. On the dunce hat. Topology, 2:341-358, 1964.

215 Carnegie, Mathematics Department, Syracuse University, Syracuse, Ny 13244, USA

E-mail address: eakofor@syr.edu

215 Carnegie, Mathematics Department, Syracuse University, Syracuse, Ny 13244, USA

E-mail address: lvkovale@syr.edu 